\title{
Unmanned Aircraft Get Together The LAPSE-RATE Campaign
}

Members of the international community developing unmanned aircraft for atmospheric research conducted a highly successful coordinated flight week in Colorado during the summer of 2018 to collect measurements, develop capabilities, and enhance community in the field.

Adapted From "Development of Community, Capabilities, and Understanding through Unmanned Aircraft-Based Atmospheric Research: The LAPSE-RATE Campaign", by G. de Boer (CIRES and NOAA), C. Diehl, J. Jacob, A. Houston, S. W. Smith, P. Chilson, D. G. Schmale III, J. Intrieri, J. Pinto, J. Elston, D. Brus, O. Kemppinen, A. Clark, D. Lawrence, S. C. C. Bailey, M.P. Sama, A. Frazier, C. Crick, V. Natalie, E. Pillar-Little, P. Klein, S. Waugh, J. K. Lundquist, L. Barbieri, S. T. Kral, A. A. Jensen, C. Dixon, S. Borenstein, D. Hesselius, K. Human, P. Hall, B. Argrow, T. Thornberry, R. Wright, and J. T. Kelly. Published online in BAMS, May 2020. For the full, citable article see DOI:10.1175/BAMS-D-19-0050.1. 

Deep convection initiation: Thunderstorms routinely form over the mountains surrounding the SLV or advect into or form over the central valley. The initiation and maintenance of storms within the central valley could be impacted by local sources of potential energy and/or coherent circulations tied to surface type gradients. Teams distributed across the northern valley measured the thermodynamic state and its temporal evolution in detail across a variety of spatial gradients to assess whether the surface plays a role in the development and evolution of storms over the valley.

Aerosol properties: Routine profiling of the lower atmosphere allowed teams operating aerosol samplers to document particle sizes and concentrations under a variety of boundary layer and synoptic wind regimes.

Valley drainage flows: Several smaller valleys feed into the SLV. Based on numerical simulations and reports from valley crop dusters, UAS were deployed to verify that clear nights could result in distinct density currents from these smaller valleys into the main SLV.

Atmospheric turbulence profiling: Research teams deployed a variety of measurement platforms to understand atmospheric turbulence in the lower atmosphere over the SLV.

\section{Campaign overview}

Ten flight teams deployed 34 different aircraft making 1,287 flights that collectively sampled altitudes between the surface and 914 MSL. One of a number of surface-based sensing systems was the University of Oklahoma Collaborative Lower Atmospheric Profiling System (CLAMPS). In addition to a surface meteorological station, CLAMPS includes a scanning Doppler wind lidar, an Atmospheric Emitted Radiance Interferometer (AERI), a microwave radiometer (MWR), and a ground station for rawinsonde releases, enabling collection of continuous wind, temperature, and humidity profiles for the entire campaign. Temperature and water vapor profiles were retrieved with a temporal resolution of $10 \mathrm{~min}$.

Beyond these systems, instrumented vehicles included mobile mesonet vehicles from the University of Nebraska-Lincoln and the NOAA National Severe Storms Laboratory, supplemented by radiosonde data. The University of Colorado also provided the recently developed Mobile UAS Research Collaboratory (MURC) to serve as a central base of operations in the field. The MURC is an instrumented van

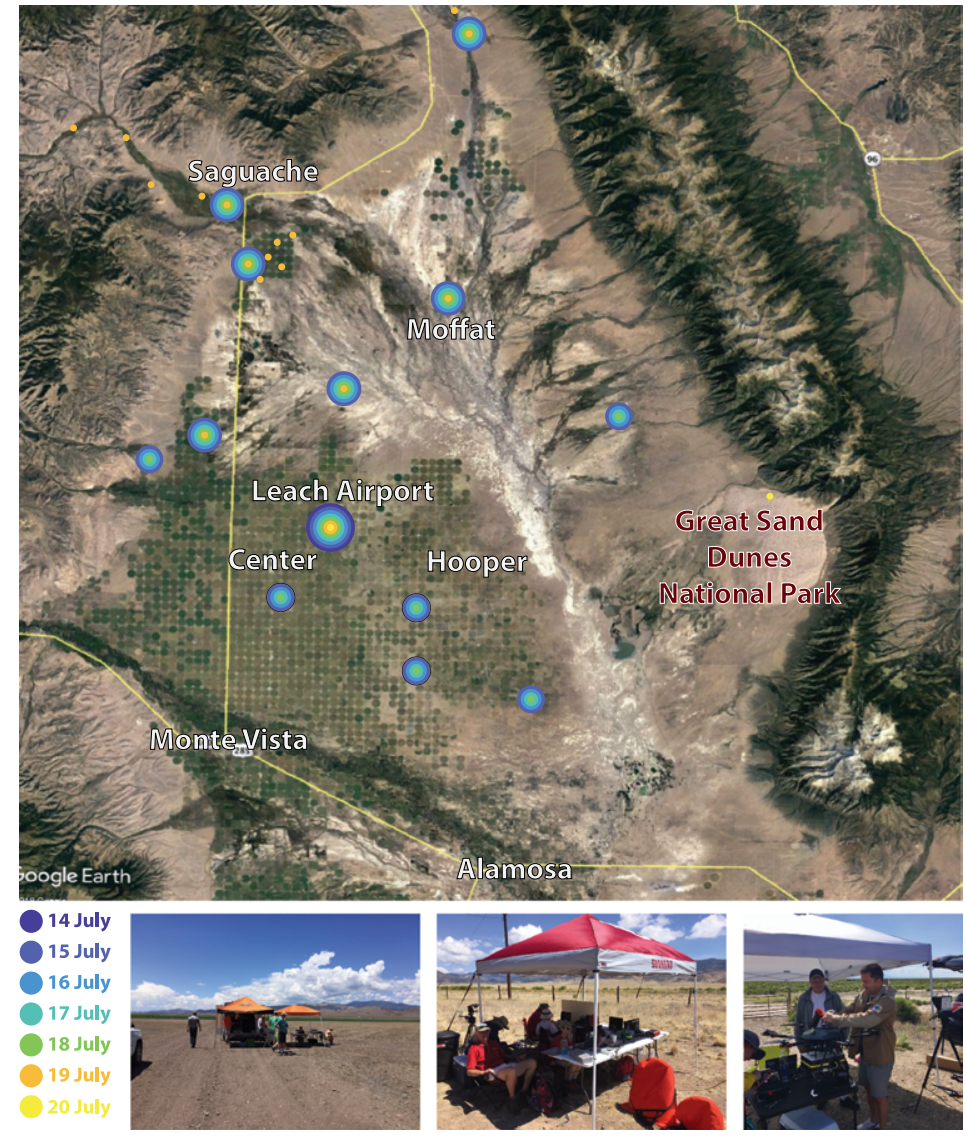

* (top) operational sites (satellite map courtesy of Google). Colors indicate the date of flights at a given location, while the size of the dot indicates the total number of days sampled at that location. (bottom) Field camps for (left to right) Oklahoma State University, University of Oklahoma, and FMI/Kansas State University teams. that supports routine computing tasks and intensive data processing and storage. Furthermore, the LAPSE-RATE team developed distributed sampling plans across the SLV based on likely weather conditions.

Weather was generally favorable during LAPSE-RATE. With a cold front north of the SLV on 14 and 15 July, monsoonal moisture advected from the Pacific. As a result, widespread afternoon thunderstorms developed over surrounding mountains, with some storms advecting over the valley.

These two days were dedicated to sampling the initiation of deep convection over a variety of surfaces. A number of groups took the opportunity to assess new vertical profiling capabilities and make additional intercomparisons. Many flights were aimed at evaluating the morning evolution of the lower atmosphere. To continue such sampling, the penultimate day of the campaign was specifically deemed a morning boundary layer transition day, with all teams strategically distributed throughout the valley to profile the lower atmosphere regularly. On 19 July, the final coordinated flight 

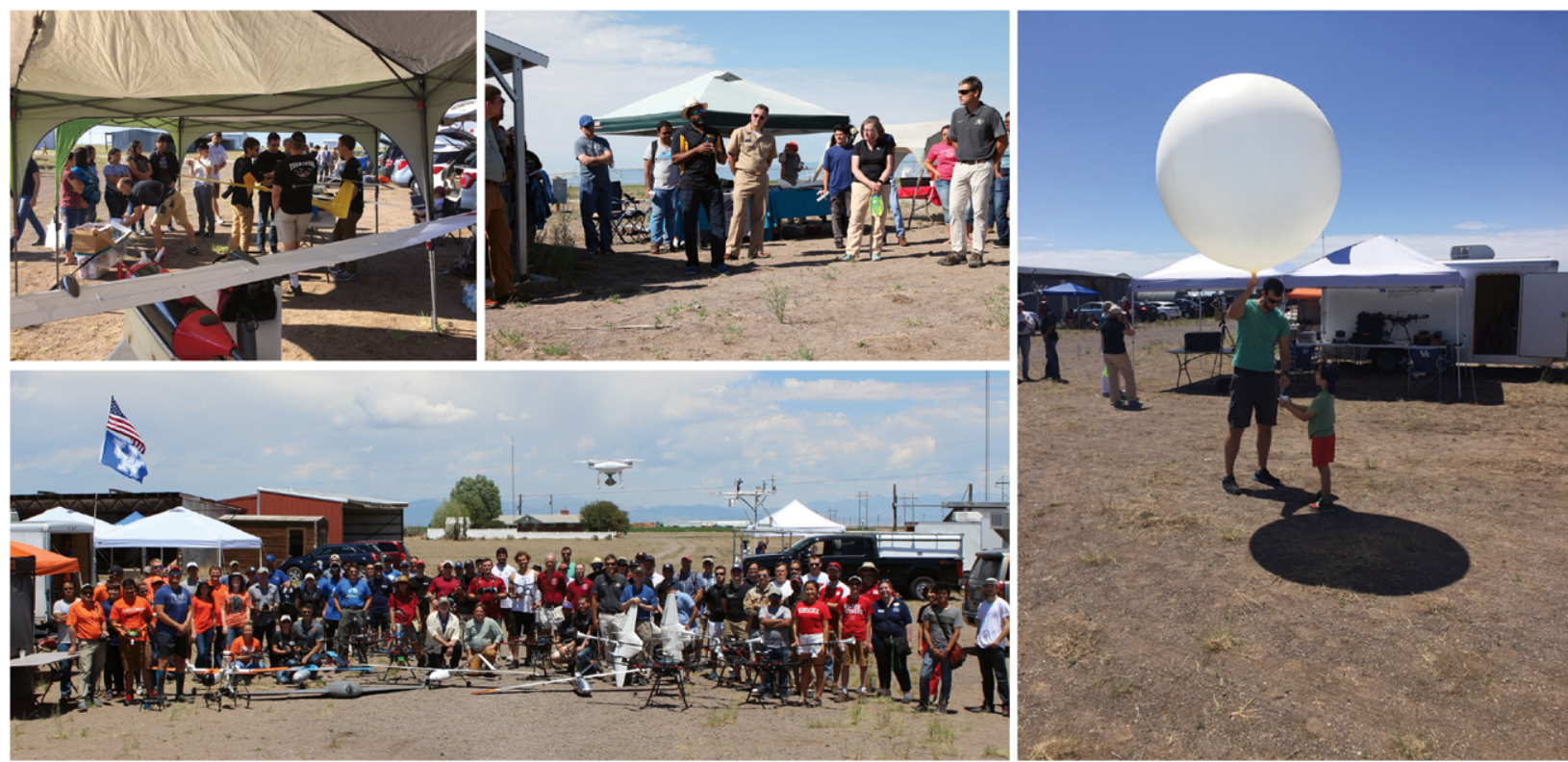

day of LAPSE-RATE, teams were redistributed to focus on measuring cold air drainage from nighttime cooling at the two smaller valleys feeding into the northern end of the SLV.

\section{Development of community}

In such a large field activity, success rests on the ability of the groups to effectively interface with each other and with supporting entities. Such coordination was supported by the ISARRA community, active funded research projects, and ongoing work by UAS Colorado, a local nonprofit. At the 2018 AMS Annual Meeting, representatives from most of the participating teams, as well as community and government organizations such as NCAR and NOAA, laid the foundation for LAPSE-RATE and initiated the coordination necessary for a successful campaign.

In addition to the precampaign coordination meetings, a small team of participants conducted a precampaign scouting trip to the SLV in May 2018. The success of both the planning discussions and the scouting trip was due in part to the integration of early-career researchers.

Finally, it was important to generate goodwill and cooperation among residents of the SLV. Toward that end, on a Community Day SLV residents were introduced to the scientists and could see and learn about UAVs and the expected benefits of LAPSE-RATE. This allowed property owners a chance to meet pilots before
A Community Day, including (clockwise from top left) Upward Bound students getting an up-close look at aircraft; panelists talking about UAS work in atmospheric science and answering community questions; a young meteorologist in the making; and LAPSE-RATE participants from most participating institutions and their aircraft. flight operations commenced. About 100 community members attended.

\section{Development of capabilities and understanding}

New platforms and sensors were deployed in the field for the first time during LAPSE-RATE. It was the first field deployment of the MURC, and for several aircraft including Kentucky's S-1000, the CU Mistral, and the EngeniousMicro LLC IntenseEye V2. It was also the first field deployment for airborne sonic anemometers mounted on rotorcraft, the University of Nebraska's thermodynamic payload, and differential temperature sensors deployed by EngeniousMicro LLC. Similarly, teams gained experience with new flight patterns and capabilities. For example, while some groups (e.g., University of Colorado) had significant experience operating aircraft in the "Follow-Me" mode, where the pilot and spotting team are in a moving vehicle and the aircraft stays close, other groups were conducting initial flights in this mode.

Having various platforms enabled observations of interesting phenomena. For example, the Finnish Meteorological Institute's PRKL1 platform carried a particle measurement module that tracked aerosol concentrations. In conjunction with other platforms measuring thermodynamic state and ground-based radar systems, such particle measurements could help to explain aerosol-cloud interactions in convective systems. 

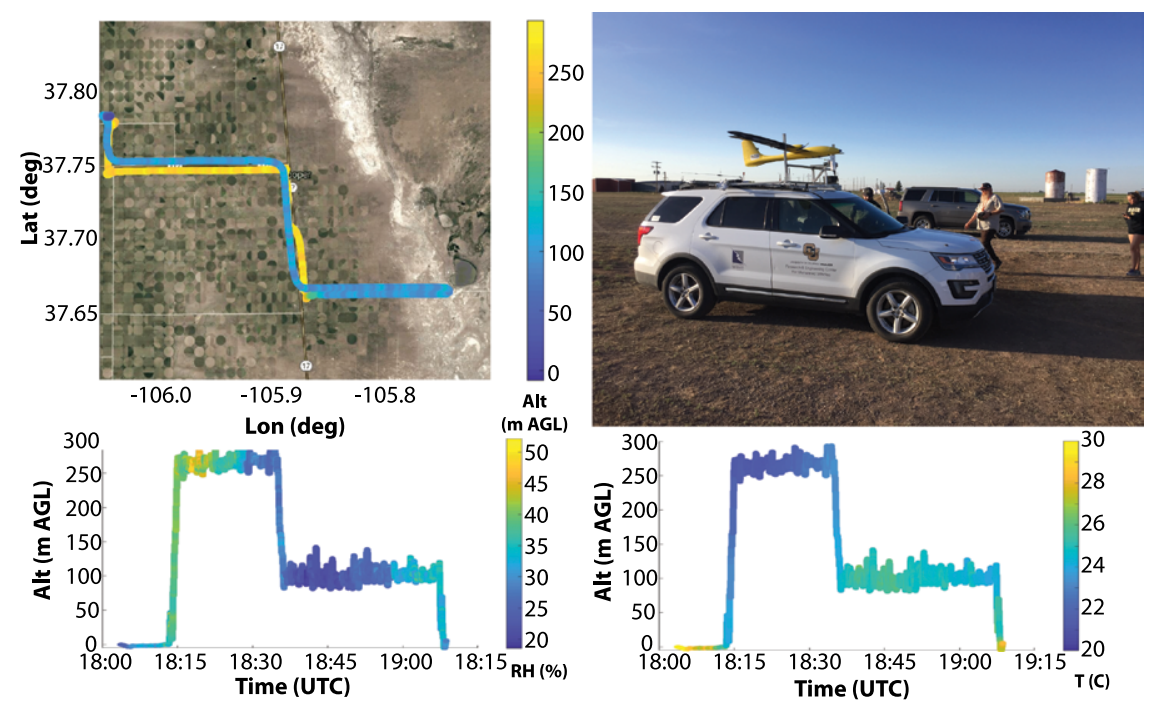

$4 *$ An example "follow me" flight from LAPSE-RATE. (top left) Track of the aircraft and ground vehicle, with color representing the aircraft altitude. (bottom) Time-height cross sections from this flight, with color representing measured (left) relative humidity and (right) temperature. (top right) University of Colorado team preparing for a "follow me" flight, with the aircraft on the roof-mounted launcher prior to takeoff.
Finally, in addition to measurement capabilities, LAPSE-RATE demonstrated a highresolution numerical model, NCAR's real-time version of the WRF Large Eddy Simulation (WRF-LES), for predicting finescale weather features in support of small UAS operations. WRF-LES was configured to run in real time on the Cheyenne supercomputer at NCAR. During LAPSE-RATE, the system was run twice daily to support next-day and day-of mission planning.

In addition to evaluating model performance, several experiments are looking at the impacts of assimilating UAS-collected data into the outer domain of the NCAR forecast system. While initial results are encouraging, studies are underway to more fully assess the value of
UAS data assimilation using more sophisticated approaches such as 3DVAR, ensemble Kalman filtering (EnKF), and hybrid techniques.

\section{Looking ahead}

All of the datasets are being processed and prepared for public consumption. For example, campaign organizers are developing a special issue in Earth System Science Data. This issue will include documented measurements obtained during LAPSE-RATE, with detailed information on sensors, platforms, deployment locations, challenges and problems, measurement uncertainty, and dataset availability associated with UAS research. The issue is intended to set an example for future (top) Wind speed and * (bottom) wind direction as simulated by WRF for the morning drainage flow at Saguache airport on 19 July 2018. Included are simulation results (lines) and observations from surface instrumentation at the Saguache airport (dots).

Simulation results are shown for experiments with

(DA) and without (No DA) assimilation of data from UAS operating nearby.

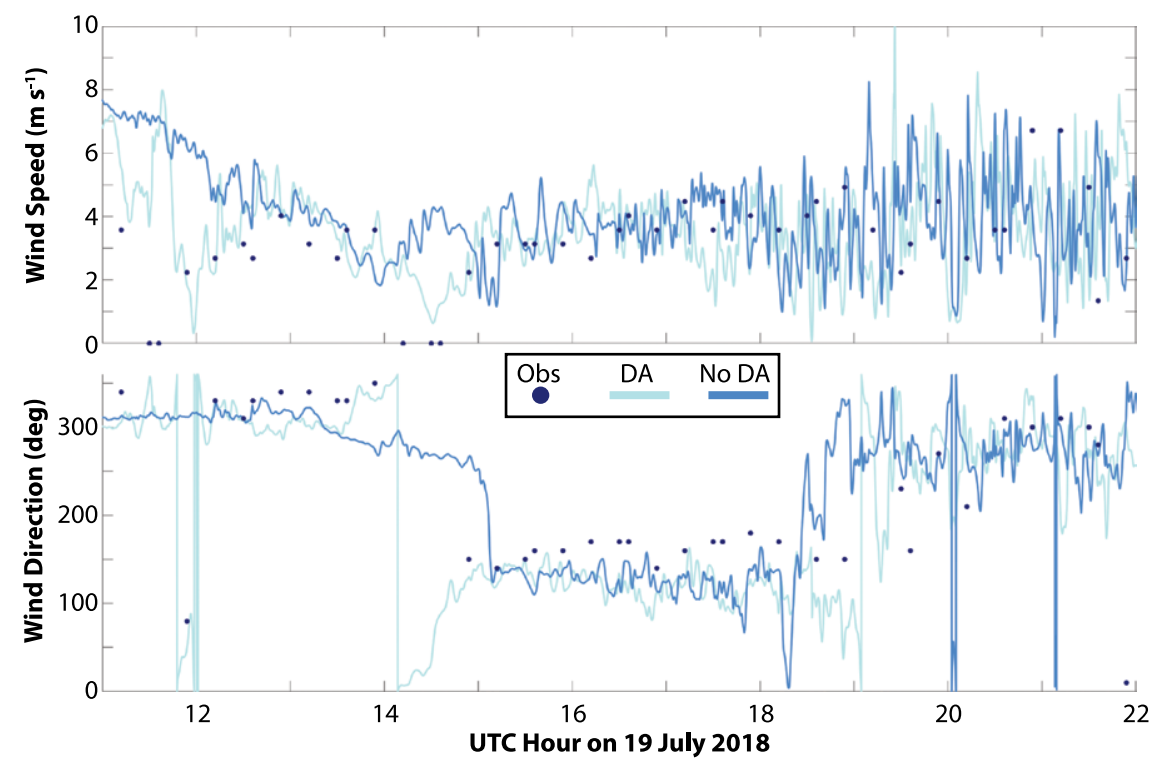


articles when the UAS-centric atmospheric science community obtains new datasets.

In addition to safely completing thousands of flight hours in the national airspace, one of the great successes coming from the ISARRA community is the development of new collaborative relationships between participants. The LAPSE-RATE campaign provides a blueprint for successful future campaigns and demonstrates the power of bringing teams with common goals together. Future ISARRA flight weeks will help to expand the atmospheric science community leveraging the many platforms used in LAPSE-RATE and further demonstrate the value of UAS-based measurements for advancing our understanding of the atmosphere.

\section{$\equiv$ METADATA}

BAMS: How did you become interested in unmanned aviation?

\section{Gijs de Boer (CIRES and NOAA): In} 2011 I worked with a small group on a review article about our knowledge of mixed-phase clouds in Arctic environments. We took a good look at critical observational deficiencies, and I began to realize that many of the gaps involved a lack of in situ information, quantities that I thought could be measured by small platforms. This sent me down the road of investigating whether UAS could offer the necessary insight.

BAMS: What are some of the shared problems revealed by working together-as in LAPSE-RATE-with other UAV teams?

GdB: There are common problems at a variety of levels. For example, accurate wind sensing has proven challenging, and we've definitely worked together to improve wind estimation. Additionally, different modes of operation, understanding which sensors are good and which are not, and sensor placement are all examples of how the community has worked together to lift up the quality of measurements from all platforms.

BAMS: What are the most surprising lessons from LAPSE-RATE?

GdB: I think that the continued rapid progression of the technology and the innovation in UAS-based atmospheric research is impressive.
Some of the tools deployed during LAPSE-RATE in 2018 have already been significantly improved upon.

BAMS: What are some examples of this more recent UAV improvement?

GdB: Everything continues to get smaller and lighter. Aircraft have become even more reliable, and instrumentation has continued to be scrutinized to improve data quality. Battery technology has also continued to improve, allowing for longer flight times and more complex missions. Yet, we have so much more to do with respect to integrating our measurements into mainstream atmospheric research.

BAMS: What are some challenges to doing more to integrate UAVs into research?

GdB: Primarily, our UAV research community is working to demonstrate the reliability and accuracy of our measurements and platforms. This is critical to having them accepted in the community. There are also some other challenges associated with airspace access and development of infrastructure to interface these observations in both mainstream research and operations.

BAMS: It seems like there's been success in this mainstreamed usage of UAS.

GdB: Campaigns like LAPSE-RATE have paved the way for UAS to be more thoroughly included in larger field campaigns. A nice example is the recent ATOMIC (Atlantic Tradewind Ocean-Atmosphere Mesoscale Interaction Campaign) and EUREC4A (Elucidating the role of clouds-circulation coupling in climate) field campaigns, where three different UAS teams were involved, and UAS were operated alongside manned research aircraft and in support of a much larger effort.

BAMS: What was the biggest challenge you encountered in the field campaign?

GdB: To be honest, LAPSE-RATE was one of the most challenge-free UAS field campaigns that I have ever been involved with. The weather was good, the teams were positive, the flight permissions were in place, the community and local officials were welcoming, and the UAS platforms generally worked as designed.

BAMS: So, then, how about in the follow-up after the field phase?

GdB: Since the campaign wrapped up, we have been working to process our datasets, document them, and share them publicly. This has gone more slowly than I imagined, and perhaps the biggest challenge has been keeping people motivated to complete these important steps. However, we're making progress and I am proud of the team for all of the work that was completed. 
AMS titles now

available as eBooks

at springer.com

\section{AMS BOOKS}

\section{RESEARCH APPLICATIONS HISTORY}

www.ametsoc.org/amsbookstore

Springer

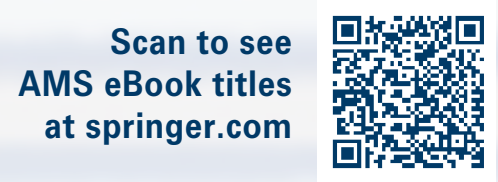

Springer

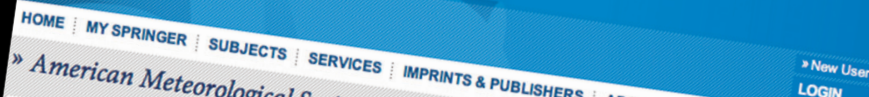

"American Meteorological SOCiety
"AOMTIN

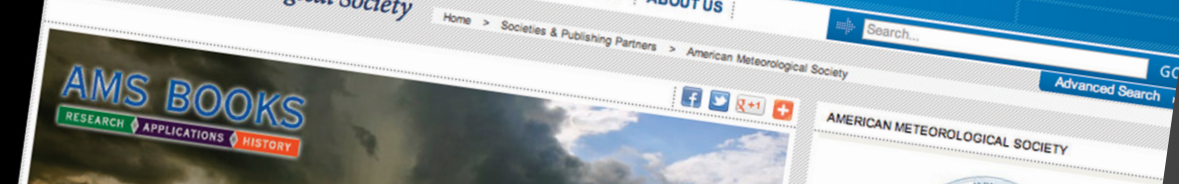

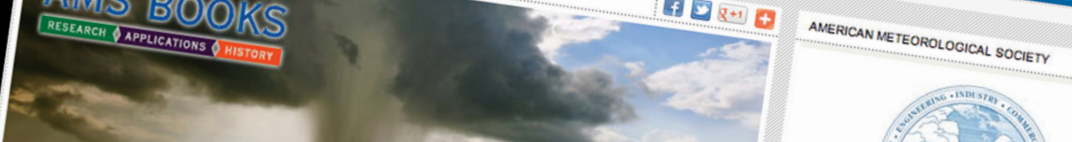

American Meteorol

Promoting the dewerogical Society

the atmos the deverap

the atmospheric and relont and disseminatb of

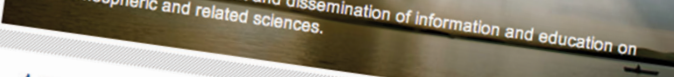

American Meteorological Society eB

AMS Books

published by the AMs a monograph publishing prent

shing program related to ho poer-

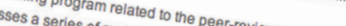

related to the as eBooks) as weil ar ariy out-ortprint vol metoorological and historica journals

to the atmospheric sciences a growing list of academis, which have been urough ographs

ind policy, and general-interest books

Sort listing by:

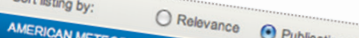

वध्ये Exporit to $\mathrm{CSO}$

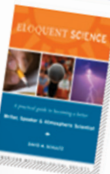

Eloquent Science

Bloquent Scie

A Practical Guite

Schulta, David
2009

Price from $\$ 45.00$

copyright Year

Avalobe

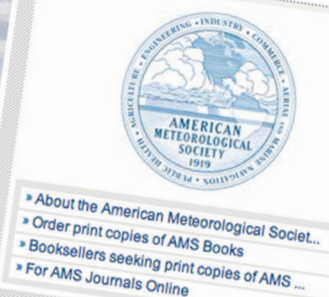

RESOURCES

Stay Informed

Receive notitication of now releases from
Books

Sub

AMS 